\title{
DESIGN AND PROCESS DEVELOPMENT FOR SMART PHONE MEDICATION DOSING SUPPORT SYSTEM AND EDUCATIONAL PLATFORM IN HIVIAIDS-TB PROGRAMS IN ZAMBIA
}

\author{
M. Willig ${ }^{1}$, M. Sinkala, R. S. Sadasivam ${ }^{3}$, M. Albert, L. Wilson ${ }^{4}$, M. Mugavero ${ }^{1}$, \\ E. Msidi, S. Brande ${ }^{5}$, J. Nikisi ${ }^{6}$, J. Stringer ${ }^{9}$, I. Tami $^{7}$, G. Henostroza ${ }^{1,9}$, \\ V. Gathibhande ${ }^{8}$, J. Menke ${ }^{9}$, M. Tanik ${ }^{8}$, S. Reid ${ }^{1,9}$ \\ ${ }^{1}$ Department of Medicine, Division of Infectious Diseases, UAB \\ ${ }^{3}$ Division of Health Informatics and Implementation Science, University of \\ Massachusetts Medical School \\ ${ }^{4}$ Department of Family/Child health and Caregiving, School of Nursing, UAB \\ ${ }^{5}$ Department of Chemistry, UAB \\ ${ }^{6}$ Jhpiego - Johns Hopkins University affiliate, Lusaka, Zambia \\ ${ }^{7}$ Department of Epidemiology, School of Public Health, UAB \\ ${ }^{8}$ Department of Electrical and Computer Engineering, UAB \\ ${ }^{9}$ Centre for Infectious Disease Research in Zambia (CIDRZ)
}

\section{UAB - University of Alabama at Birmingham Birmingham, Alabama 35294}

\begin{abstract}
The widespread adoption of cell phones and other mobile platforms represents an opportunity to extend the benefits of personalized, point-of-care, healthcare applications to providers and patients in the developing world. However, the challenges facing the effective deployment of mobile health care applications are complex, and thus require a scalable, flexible, and configurable approach. A service-oriented-architecturebased conceptual framework is proposed to address the challenges of developing and deploying mobile health care applications. A particular emphasis of the framework is a service-agent-modeling-based composite process-personalization support that is needed to support the diverse and adaptable needs of the users.
\end{abstract}

\section{INTRODUCTION}

Once thought impossible, the dedication and efforts of countless international organizations and individuals have made HIV/AIDS therapy available in developing nations where it is estimated $90 \%$ of global cases are present. Despite the vastly improved access to antiretroviral therapy (ART), in 2007 World Health Organization (WHO) estimates indicated only 31\% of those infected in low- and middle income countries had accessed HIV/AIDS therapy (Munjanja et al, 2005; PEPFAR, 2009; WHO, 2006). Reduced human resources are a key barrier to the provision of HIV care in resource limited settings. Contributing factors include a limited supply of new healthcare workers coming into the workforce, inadequate human resource management systems for recruitment, deployment and retention, attrition due to HIV/AIDS, limited career and professional opportunities, and increasing rates of international migration (Munjanja et al, 2005; WHO 2008a; WHO 2008b). In response to these shortages, and to maximize available human resources, the World Health Organization (WHO) published guidelines related to task-shifting, the strategy of moving tasks from highly qualified health workers to workers with shorter duration training needs. Alongside task-shifting, the WHO stresses the need for efforts to increase the overall number of trained healthcare workers and to establish appropriate quality assurance mechanisms to evaluate and monitor clinical outcomes.

HIV care has become increasingly complicated due to a number of factors. The complexity of dosing antiretroviral therapy continues to expand as more agents are added to the treatment arsenal and older drugs are coformulated into new combinations. Providers are prone to various dosing errors if they fail to account for multiple patient characteristics at the time of prescription (Purdy et al., 2000; Rastegar et al., 2006). At the University of Alabama at Birmingham HIV care clinic, we found dosing errors occurred in $6 \%$ of all prescriptions and in 31\% of those in patients with renal dysfunction (Willig et al., 2007). Dosing errors may lead to increased drug toxicity, which in turn may adversely 
affect adherence to therapy and hasten the development of drug resistant virus and regimen failure, which impacts HIV/AIDS morbidity and mortality. In the developing world, having to move from a first line regimen to a second line regimen increases the cost of ART tenfold (Sukasem et al., 2007; Sungkanuparph et al., 2007; Marconi et al., 2008). At present, third line regimens do not exist, meaning the development of resistance to second line regimens leaves patients without therapeutic options.

An additional challenge is that patients with advanced HIV/AIDS often need concomitant treatment for co-existing opportunistic infections such as tuberculosis (TB). Agents used to treat TB often interact with drugs used to treat $\mathrm{HIV}$, resulting in changes in serum levels that may contribute to toxicity (elevated drug levels) or treatment failure (lowered drug levels) (Onyebujoh et al., 2007; Lalloo and Pillay, 2008; Scano et al., 2008; Manosuthi et al., 2009). Faced with the challenge of appropriately dosing complex ART and TB regimens, providers would benefit from targeted informatics tools that would assist the prescription of appropriately dosed therapy. We are proposing to develop such a tool in collaboration with front line care providers in Zambia. We propose the utilization of mobile phone technology due to its penetration, ease of use and familiarity to users, and its potential to harbor software. Such a mobile phone system will not only allow for dosing decision support at the point of care, but it will also serve as a platform for the dissemination of continuing medical education, and enhance the skills of care providers longitudinally. Such a strategy holds the promise of helping patients by decreasing toxicity and maximizing effectiveness, as well as providing a practical tool that can monitor quality assurance. Monitoring patterns of prescription error can provide insight into areas where educational intervention is needed. What follows is a summary of a staged approach for the development of such software. We anticipate that such expert dosing software could play an important role in minimizing dosing errors, monitoring quality of care, and targeting educational interventions as task-shifting initiatives are increasingly implemented throughout the world.

\section{REQUIREMENTS}

The goal is to create a flexible software tool that could be utilized at the front lines of HIV and TB care in resource limited settings. The tool must be:

User friendly: The tool should provide clear value to providers. The input of patient and planned treatment information should be simple, and result in readily interpretable and patient specific dosing recommendations. To accomplish this goal, patient specific characteristics (i.e. height, weight, pertinent laboratory values, etc.) will need to be entered before a planned treatment is formulated. The application will then calculate and present individualized treatment and dosing recommendations based on patient characteristics.

Flexible: It must allow for easy and accurate capture and storing of this information in a central database for later access. Though much data (i.e. drug dosing recommendations) will be portable across settings, the application must be flexible enough to allow for some individualization of treatment recommendations based on the needs and available drugs in specific settings.

Accessible: The application needs to be readily deployable in resource rich and resource limited settings. Due to their penetration and rapidly increasing computing power, we feel mobile phones provide an optimal platform for dissemination of this application. The application could be installed on mobile phones and provide recommendations even at times without ready access to phone networks. Educational modules could be automatically downloaded to provider phones when in a service area, to be accessed and completed later. Evaluations of these modules would be uploaded automatically, and scores sent back to providers once they return to service areas. In this fashion, one can envision each HIV treatment program establishing continuing education requirements and have a platform in place to readily disseminate such interventions. Likewise, in case it becomes necessary to communicate to providers new scientific findings that would have an impact on current practice, a network to send and track review of these data would be in place. This new network might also be used to disseminate information on new epidemics or other public health emergencies.

Prepared for additional linkage: At the central repository for information, individual patients will have a system wide identifier (primary key), around which the data structures will be organized. By structuring the data this way, the toolset may later be able to link to and display information captured throughout the healthcare system (laboratory, medication history/changes at other sites, dates/times/locations of previous visits, etc). Laying the foundation to be able to potentially organize and later share information from a central site will allow future healthcare providers to have reliable information on past care that patients have received. For example, such thorough capture of historical information will enable providers to make optimal treatment decisions based on comprehensive knowledge of past medication exposure. It has been reported that as many as $40-50 \%$ of patients are lost to follow up from where they began to 
Integrated Design and Process Technology, IDPT 2009

Printed in the United States of America, November, 2009

(C) 2009 Society for Design and Process Science

receive HIV care in developing nations. Loss to follow up is associated with poor treatment outcomes and increased mortality. The capture of the proposed data through a national network of mobile phones could provide insight into patient histories across multiple sites of care. These data in turn could help guide the development of interventions to mitigate the risks of "losing" patients and ultimately improve HIV care outcomes. Laying the foundation to cross link to other databases from the very beginning will lay the groundwork for potentially expanding the functionality of this application in the future.

\section{FUNCTIONAL REQUIREMENTS}

Initially, this application will provide point of care assistance in the dosing of antiretroviral and tuberculosis therapy. This will only represent the first stage of application development, with further phases and correspondingly expanded functionality to follow. Below we propose a "growth plan" for the application, the development of additional functionality seeking to respond to the most pressing needs of our partners.

Stage 1: Provision of educational materials. The mobile application will allow the display of documents such as national guidelines for the treatment of HIV and/or TB therapy. These will be updated and sent to providers periodically as soon as new iterations become available. Continuing medical education modules which test providers on their knowledge of important changes to treatment recommendations could be delivered as well. This will allow national programs ready access to a distance education network that will potentiate their ability to disseminate needed guidelines in a timely fashion to the field.

Stage 2: Decision support for adult dosing recommendations for HIV and/or TB therapy. Guided by comprehensive review of drug prescribing information, a set of rules governing the appropriate dosing of adult HIV and TB therapy will be completed. These rules will include the detection of interactions between therapies for illnesses that could compromise drug levels to the detriment of those under care (high drug levels leading to toxicity, or low drug levels leading to loss of effectiveness). The clinical parameters that need to be taken into account in the dosing of each drug will be entered into a streamlined form. After entry, providers will be asked to select the planned therapeutic regimen. Upon completion, the application will generate dosing recommendations for the relevant drugs for the specific patient. In this manner, patient specific dosing will be calculated taking into account such factors as weight, CD4 value, creatinine clearance, coadministration of HIV and TB therapy.

Stage 3: Decision support for pediatric dosing recommendations for $\mathrm{HIV}$ and/or TB therapy. Dosing TB and HIV therapy in children represents additional challenges. The addition of the patient characteristics needed to generate appropriate dosing recommendations for HIV and TB infected children will be included. Decision support taking into account the relevant clinical characteristics will be generated at the point of care.

Stage 4: Addition of drug interactions. We will extend the capabilities of the application to provide guidance on drug interactions covering all potential medications the patient could be using. The average life expectancy of HIV positive individuals is calculated to be approximately 70 years of age. As our HIV infected population ages and requires additional medication for other concomitant medical conditions (e.g., cardiac disease or diabetes), the ability to easily and reliably check for drug interactions with the existing HIV treatment regimen will increase in importance. We will explore standalone applications to add such functionality, and work with strategic partners or knowledge vendors to acquire databases that can be used for this purpose.

Stage 5: Linkage to existing databases and central information repository. Herein lies the potential to expand the utility of this application in the future. An example would be linkage to existing national laboratory databases that would allow access to laboratory data on all patients wherever providers have access to a mobile phone network. If one were to add linkage to patient HIV drug resistance reports, it will greatly aid in the selection of optimal new regimens. A consequence of the expansion of the use of mobile applications to receive feedback on appropriate dosing is that the recommended regimens could be stored in the central database along with stop dates. When another provider in a different geographic setting is starting subsequent therapy, a record of the initiation dates and agents utilized in the past would be available. Currently, a "history of prior agents" often depends exclusively on patient recollection. The ability to have access to well characterized past regimen data will present health care providers with comprehensive drug histories that will allow for the initiation of optimal subsequent therapy.

\section{LOOKING AHEAD}

A mobile phone application, well adapted and appropriately implemented in daily clinical practice in the targeted settings, will enable providers to potentially avoid erroneous prescription of HIV and TB therapy. 
Integrated Design and Process Technology, IDPT 2009

Printed in the United States of America, November, 2009

(C) 2009 Society for Design and Process Science

The establishment of a national network of providers using such an application will allow health ministries to disseminate guidelines and complete continuing medical education initiatives in rapid fashion. Use of tools, such as the application described, have the potential to allow for monitoring of HIV and TB outcomes for healthcare providers serving patients as part of task-shifting initiatives. These data will be necessary to evaluate the effectiveness of task-shifting initiatives, as well as allowing the detection of issues that will help to target continuing medical education for such providers. Building a system with the foundation in place to capture the data that will allow for the optimal selection of future therapy in resource limited settings will serve to provide reliable databases that will improve longitudinal patient care. This will be critical in HIV therapy where the current paradigm calls for lifelong ART, and subsequent treatment decisions are influenced by prior treatment and resistance history. Due to the expanding computing capacity, increased penetration of mobile phone networks and newer alternatives such as solar-powered cell phones, this technology allows for the deployment of software tools in remote settings where comprehensive informatics resources are lacking. Through carefully crafted software applications, we can bring many of the benefits of medical informatics to resource limited settings.

\section{REFERENCES}

Lalloo, U. G. and Pillay, S., 2008, "Managing tuberculosis and HIV in sub-Sahara Africa," Curr HIV/AIDS Rep, Vol. 5, Iss.(3), pp. 132-9.

Manosuthi, W., Sungkanuparph, S., Tantanathip, P., et al., 2009, "A randomized trial comparing plasma drug concentrations and efficacies between 2 nonnucleoside reverse-transcriptase inhibitor-based regimens in HIVinfected patients receiving rifampicin: the N2R Study," Clin Infect Dis, Vol. 48, Iss.(12), pp. 1752-9.

Marconi, V. C., Sunpath, H., Lu, Z., et al., 2008, "Prevalence of HIV-1 drug resistance after failure of a first highly active antiretroviral therapy regimen in KwaZulu Natal, South Africa," Clin Infect Dis, Vol. 46, Iss.(10), pp. 1589-97.

Munjanja, O. K., Kibuka, S., \& Dovola, D., 2005, "The nursing workforce in sub-Saharan Africa", Geneva, Switzerland: International Council of Nurses, accessed February 3, 2008, <http://www.icn.ch/global/Issue7SSA. pdf>

Onyebujoh, P. C., Ribeiro, I. and Whalen, C. C., 2007, "Treatment Options for HIV-Associated Tuberculosis," J Infect Dis, Vol. 196 Suppl 1, pp. S3545.
PEPFAR, "President's Emergency Plan for AIDS relief", accessed July, 20 2009, <http://www.pepfar .gov>.

Purdy, B. D., Raymond, A. M. and Lesar, T. S., 2000, "Antiretroviral prescribing errors in hospitalized patients," Ann Pharmacother, Vol. 34, Iss.(7-8), pp. 8338.

Rastegar, D. A., Knight, A. M. and Monolakis, J. S., 2006, "Antiretroviral medication errors among hospitalized patients with HIV infection," Clin Infect Dis, Vol. 43, Iss.(7), pp. 933-8.

Scano, F., Vitoria, M., Burman, W., et al., 2008, "Management of HIV-infected patients with MDR- and XDR-TB in resource-limited settings", Int J Tuberc Lung Dis, Vol. 12, Iss.(12), pp. 1370-5.

Sukasem, C., Churdboonchart, V., Chasombat, S., et al., 2007, "Prevalence of antiretroviral drug resistance in treated HIV-1 infected patients: under the initiative of access to the NNRTI-based regimen in Thailand", $J$ Chemother, Vol. 19, Iss.(5), pp. 528-35.

Sungkanuparph, S., Manosuthi, W., Kiertiburanakul, S., et al., 2007, "Options for a second-line antiretroviral regimen for HIV type 1-infected patients whose initial regimen of a fixed-dose combination of stavudine, lamivudine, and nevirapine fails", Clin Infect Dis, Vol. 44, Iss.(3), pp. 447-52.

Willig, J. H., Westfall, A. O., Allison, J., et al., 2007, "Nucleoside reverse-transcriptase inhibitor dosing errors in an outpatient HIV clinic in the electronic medical record era", Clin Infect Dis, Vol. 45, Iss.(5), pp. 658-61.

WHO, 2006, "Treat, train, retain. The AIDS and health workforce plan. Report on the consultation on AIDS and human resources for health", Geneva, Switzerland, World Health Organization, accessed July 20, $2009<$ http://www.who.int/hiv/pub/meetingreports/ TTRmeetingreport2.pdf $>$.

WHO, 2008a, "Task shifting: Rational redistribution of tasks among health workforce teams: Global recommendations and guidelines", Geneva, Switzerland: World Health Organization, accessed July 20, 2009 at <http://www.who.int/healthsystems/TTRTaskShifting.pdf $>$.

WHO, 2008b, "Towards universal access: scaling up priority HIV/AIDS interventions in the health sector: Progress Report", accessed 20 July 2009, <http:// www.who.int/hiv/mediacentre/2008progressreport/en/pri nt.html>. 